\title{
Genetic structure of breeding pigs of Large White, bred in Russia
}

\author{
Getmantseva L., Bakoev S., Kostyunina O., Traspov A., Prytkov Yu., Bakoev N. \\ Federal Science Center for Animal Husbandry named after Academy Member L.K. Ernst \\ Dubrovica, Russia \\ ilonaluba@mail.ru
}

\begin{abstract}
This paper presents a study of the genetic structure of breeding pigs of Russia, belonged to a Large White.

\section{Keywords - genetic structure, pig, Large White, Porcine HD}

In recent decades, the global commercial pig industry has led to significant business consolidation. Mergers and acquisitions of small genetic centers have resulted in a limited number of remaining international breeding companies [1]. Consequently, the breeding lines belonging to these companies also experienced a high degree of consolidation. In the Russian Federation, the Large White represent $54.1 \%$ of pigs [2]. The aim of the work is to study the genetic structure of the breeding pigs of the Russian Federation, which belong in the Large White breed.

The main companies supplying genetic material to the world market of pigs are Dan AvI (Denmark), Genesus (Canada), PIC (Great Britain), Hypor (Holland), Nucleus (France), Hermitage Genetics (Ireland). In our work, we investigated pigs from three Selection Genetic Centers (Farm) of Russia. The population of these Farms formed in 20122014 by imported genetics of different companies and further improved in accordance with its own strategy. The pigs from Farm1 came from Nucleus (France), Farm 2 - Dan AvI (Denmark) and Farm 3 - Hermitage Genetics (Ireland).
\end{abstract}

The study was on Large White pigs $(n=53)$ from three Farms of Russia: Farm $1(\mathrm{n}=25)$, Farm $2(\mathrm{n}=16)$ and Farm 3 $(n=12)$. Genome DNA extracted from sample (ear plucks) by a set of reagents DNA-Extra-2 (OOO Syntol, Russia). Genotyping of animals was performed using GeneSeek GGP Porcine HD (San Diego, USA), including 68540 SNPs. The functions of the NAM package [3] used to impute missed values using the machine-learning algorithm Random Forest. As a result, 46845 SNPs left for further analysis. The Principal Component Analysis (PCA) method in Plink v.1.9 [4], the ggplot2 package [5], Admixture 1.3 [6] and Ancestry Painter [7] were using the estimation of the population structure.

The results of the study showed a clear division into three clusters of Large White breed (Figure 1). Each cluster corresponded to a specific Farm. Can be clearly identified the genetic structure of the companies: Nucleus (France), Dan AvI (Denmark) and Hermitage Genetics (Ireland) from the data obtain. Among the groups studied, the most consolidated population is determined in Farm 3 based on Irish genetics. In Farm 1 and 2, along with the prevalence of French and Danish genetics, respectively, we can note the presence of a fraction of the genome of different origin, which is probably due to breeding work aimed at improving the breeding stock of pigs and possible formation of their own genetic structure.
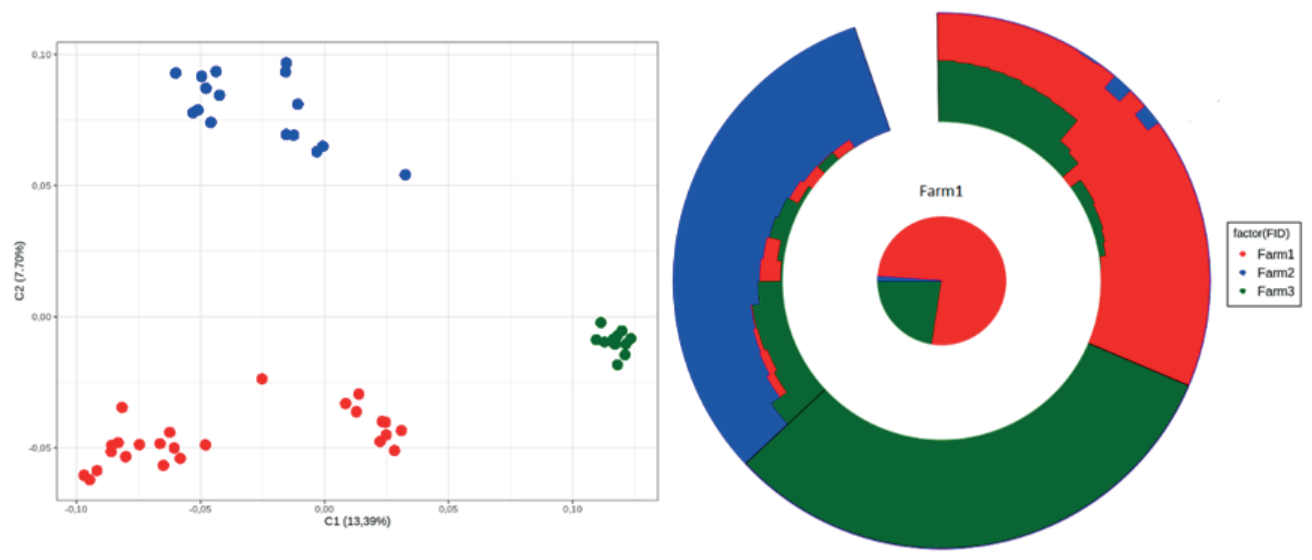

Fig. 1. Genetic structure of breeding pigs of Large White, bred in Russia

\section{REFERENCES}

[1] I. Hulsegge, M. Calus, R. Hoving-Bolink et al. Impact of merging commercial breeding lines on the genetic diversity of Landrace pigs. Genet Sel Evol 51, 60 (2019)

[2] Yearbook on pedigree work in pig husbandry on the farms of the Russian Federation M.: Publishing house FGNU VNIIplem, 2018. $192 \mathrm{p}$.

The work is funded by the RSF grant No. 19-16-00109.
[3] A. Xavier, S. Xu,W.M. Muir, K.M. Rainey NAM: association studies in multiple populations Bioinformatics. 2015. 31(23): 38623864.

[4] C.C. Chang, C.C. Chow, L.C. Tellier et al. Second-generation PLINK: rising to the challenge of larger and richer datasets. Gigascience. 2015. 25;4:7.

[5] H. Wickham. ggplot2: Elegant Graphics for Data Analysis. 2009.

[6] D.H. Alexander, J. Novembre, K. Lange. Fast model-based estimation of ancestry in unrelated individuals. Genome Research, 19:1655ไu20131664, 2009.

[7] F. Qidi. The AncestryPainter software2 is available at http://www.picb.ac.cn/PGG/resource.php 